\title{
Acknowledgement to Reviewers of Healthcare in 2019
}

Healthcare Editorial Office

MDPI, St. Alban-Anlage 66, 4052 Basel, Switzerland

Received: 15 January 2020; Accepted: 15 January 2020; Published: 15 January 2020

The editorial team greatly appreciates the reviewers who have dedicated their considerable time and expertise to the journal's rigorous editorial process over the past 12 months, regardless of whether the papers are finally published or not. In 2019, a total of 170 papers were published in the journal, with a median time to first decision of 19 days and a median time from submission to publication of 43 days. The editors would like to express their sincere gratitude to the following reviewers for their generous contribution in 2019:

\begin{tabular}{ll} 
Abdullah Al-Zaghal & Ángel Romero Martínez \\
Adam B. Edwards & Angela Yang \\
Adewuyi Ayodele Adeyinka & Anjali Zarkar \\
Adrián Ceccato & Ann Svensson \\
Agnieszka Jaworowska & Anna M. Schotthoefer \\
Aida Bairam & Anna Sołtysik-Piorunkiewicz \\
Albert Figueras & Anne Kjemtrup \\
Alberto Modenese & Annette Boaz \\
Alejandro Sanz & Anthony B. Miller \\
Alessandro Alaimo & Anthony Herbert \\
Alessandro Stefanini & Antonia Arnaert \\
Alessandro Tonacci & António Abreu \\
Alicia Peñalba & Antonio Clavenna \\
Alicja Domagala & Antonio J. Ramos-Morcillo \\
Alonço Viana & Antonio Luque \\
Alpo Vuorio & Anurag Sharma \\
Álvaro Francisco Lopes De Sousa & April D. Davis \\
Alvaro Toledo & Arifin Sandhi \\
Amany Hassan & Aristeidis H. Katsanos \\
Ambereen Mehta & Armin Dietz \\
Amosy E M'Koma & Ashish Pathak \\
Amutha Selvamani & Asrat Amnie \\
Amy Throckmorton & Assunção Nogueira \\
Ana Belen Ortega Avila & Astrid Fink \\
Andras Lakos & Athina Economou \\
Andreas Rantala & Aurelio Sessa \\
Andres R. Schneeberger & Awais Farid \\
Andrew Chetwynd & Ayoub Al-Jawaldeh \\
\hline
\end{tabular}




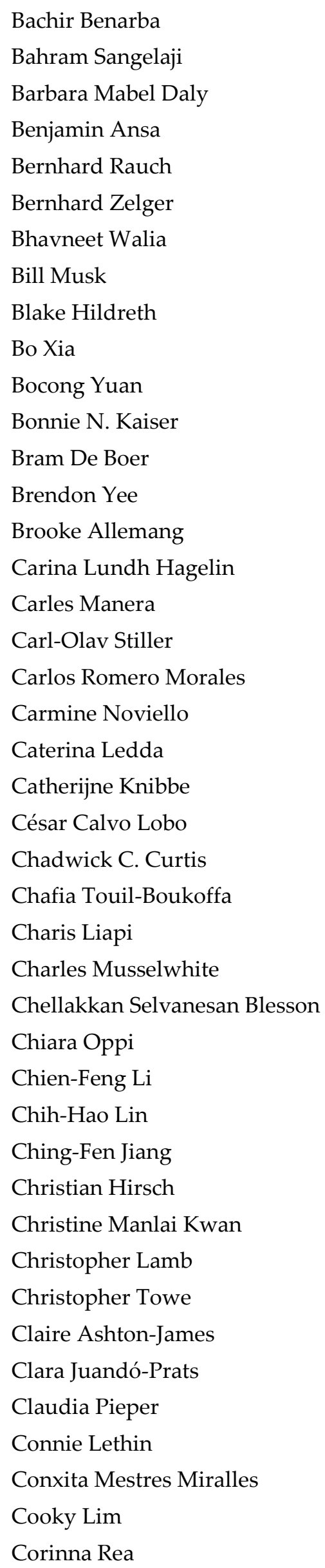

Dan Horsfall

Dan Romer

Daniel Dibaba

Daniel López-López

David Díez

David Geldmacher

David Hutchon

David Lim

David McDonald

David Naranjo-Hernández

David Zweiker

Denise Taylor

Desmond Whalen

Devayani Bhave

Diana M. Layne

Diana Layne

Diana Woods

Dirk Frans De Korne

Donald H. Shaffner

Dong-Woo Cho

E.A.M. Bulder

Edith Claros

Edmundo Rosales-Mayor

Edward Tolhurst

Egbert Oosterwijk

Elaine Stur

Elena Riza

Eleonora Nucera

Elin A. Björling

Elizabeth Nappi Corrêa

Elsa Pegado

Emma Bartfay

Emma Forsén Mantilla

Erika Di Zazzo

Evgenia Halkia

Ewa Lange

Fátima Roque

Federica Sganga

Fernando Fajardo-Bullón

Filipa F. Vale

Filippa Bono

Filomena Morisco

Finn Diderichsen 


For Yue Tso
Fotios Chatzitheodoridis
Francesca Gorini
Francesco Pistelli
Franco Muggia
Fulvio Laus
Gabriela Topa
Gaëtane Caesens
Gang Kou

Gaynell M. Simpson

Gennaro Cormio

Gernot Kronreif

Gianluca Catania

Giorgina Barbara Piccoli

Giulio Fusco

Giulio Illuminati

GOTO Yoshikazu

Grace Vincent

Gregg Nelson

Grzegorz Mentel

Guang Xu

Guangming Cheng

Guanshi Zhang

Guido Erreygers

Guruswamy Mahesh

Gustavo G. Nascimento

Gusztav Belteki

H.J. Tange

Hae-chun Rhee

Hana Morrissey

Han-Na Kim

Hans De Loof

Harishchandra Dubey

Hattie Wright

Helena Fernández López

Helge Stalsberg

Helmut Frohnhofen

Henrik Andersson

Hiroshi Kuroki

Hiroto Honda

Ho Lin

Holly Ahern

Holly B. Herberman Mash
Holton Avery

Hui Ting Chng

Hui $\mathrm{Xu}$

Hung-Chung Huang

Hyeongsu Kim

Hyun Hee Heo

Iain Scott

Ian Walsh

Ioannis E. Tsolas

Ioannis Ilias

Iole Vozza

Iracema Leroi

Isabel Castillo

Isao Otsuka

Ivo Iliev

J. Christopher Ehlen

Jacek Pyżalski

James W. Sonne

Jane Marke

Janet Mattsson

Jaume Coll-Font

Javier Ortuño Sierra

Jayoung Han

Jenil Patel

Jennifer Frediani

Jennifer L. Scheid

Jerald Dumas

Jeroen Dikken

Jeroen Van Gorkom

Jérôme Berger

Jessica McNeil

Jesús Adrián López

Jesus Prieto-Lloret

Jhinuk Chatterjee

Jia Liu

Jill Hines Bennett

Jing Feng

Jinhong Yang

Jitendra Singh

Jitka Klugarová

Joachim Storsberg

Joanne Yaffe

Joaquín Cayón De Las Cuevas 
Joel N. Maslow

Joël Wagner

Johanna H. Van Der Lee

John F. Newman

John Halperin

Johnny Holloway

Jorge H. Leitão

Jørgen Trankjær Lauridsen

JOSE ANTONIO DE PAZ

José Enrique Torres Vaamonde

José Garcia-Alonso

José Joaquín Mira

José M. Laínez-Aguirre

Jose Moran

José R. Villar

Joseph Cesarine

Joseph O. Falkinham III

Joukje Swinkels

Julie Balen

Julie McCullough

Julie Walabyeki

Jun Yasuda

Jung Tae(Steve) Kim

Jürgen Vormann

Jyotsna Shah

Kaite Yang

Karen Bishop

Karen Eisler

Karen Setty

Karl Bechter

Katarzyna Sygit

Kathleen Galvin

Kazuhiro P. Izawa

Kazumichi Fujioka

Kenneth A. Knapp

Kenneth Liegner

Kentaro Okuno

Keun Ho Ryu

Keun-Yeong Jeong

Kezia Gaitskell

Khalid El Bairi

Kimberly Jamie

Klaus Kayser
Koji Matsuo

Koji Naruishi

Kosuke Minaga

Kouichiro Kawano

Kriti Puri

Krzysztof Laudanski

Kyaien Conner

Kyle B. Walsh

Ladislav Volicer

Laima Sapezinskiene

Laiqun Jin

Laura Naegele

Laura Sbaffi

Lauretta Luck

Leonardo Juan Ramirez Lopez

Lewis Carpenter

Li-Fan Liu

Linda Baumann

Loredana Ivan

Loredana Sarmati

Lorenzo Desideri

Lorraine A. Sheppard

Louise Underdahl

Luana Nosetti

Lucia Ansani

Lucia Ramirez-Baena

Lukai Liu

Lynda Sedikki

Lynne S. Nemeth

Maddalena Lettino

Maggie Longacre

Makoto Endo

Manuel García-Goñi

Manvi Sharma

Marcel Hanisch

Marcel Olde Rikkert

Marcia E. Herman-Giddens

Marcial Velasco Garrido

Marco Dettori

María Del Carmen Pérez-Fuentes

Maria Francesca Alvisi

Maria João De Almeida Coelho De Sousa

Maria Luca 


Maria Olenick
Marianne MIddelveen
Maribel Pino
Marie B. Corkery
Marie-José H.E. Gijsberts
Mariel Colmán Martínez
Marijana Bađun
Mariusz Duplaga
Mark Dreher
Marta Tremolada
Marta Vasconcelos
Martijn Lambert
Mary Sharp
Mary W. Mathis
Maryam Malekigorji
Masafumi Nozoe
Massimiliano F. Peana
Massimo Bracci
Matteo Gulino
Matteo Moroni
Matthias Klumpp
Maurits Van den Noort
Meagen Rosenthal
Megha Sharma
Melanie Gentry
Melanie Turk
Melanie Wills
Melissa Caimano
Mellissa Withers
Michele Piana
Michele Totaro
Mihye Cho
Mika Salmi
Minh Hoang Nguyen Lee
Min-Seong Ha
Minyon Avent
Mitsushige Sugimoto
Miwa Yamamoto
Mohad Parnianpour
Milva
Maruffini
Monadin Ahmed
Mica

Munjae Lee

Muppala N. P. Raju

Nagarjuna Cheemarla

Nasser Sharareh

Nathanael Ojong

Navneet Rai

Neil Chadborn

Nelda Mier

Neveen Said

Nicholas B. DeFelice

Nicky Britten

Nicola Guess

Nicola Nante

Nicola Silvestris

Nicola Turner

Nina Flanagan

Nnaemeka Odo

Noren Hasmun

NOZAKI Kosuke

Orazio Aiello

Orly Sarid

Paiboon Jungsuwadee

Panagiotis Pentaris

Paola Lova

Patrick D. Kumavor

Patrick Marius Koga

Paul E. Lyons

Paul Egan

Paul Schoenhagen

Paul Tulkens

Pauline Marsh

Paweł Chruściel

Paweł Pławiak

Paweł Więch

Pei Ling Choo

Peter Bob Peerenboom

Peter Lee

Peter Mayne

Peyman Shokrollahi

Philippa Howden-Chapman

Pierre La Rochelle

Ping Zou

Pingping Han 


\author{
PRAKASH Kumar \\ Qihong Deng \\ Qinyi Wang \\ Quan-Hoang Vuong \\ Quentin Durand-Moreau \\ Rahim Mutlu \\ Rajeev Cherukupalli \\ Ramune Jacobsen \\ Raoul Furlano \\ Rasa Barkauskienė \\ Refaat Hegazi \\ Renée J. Bogschutz \\ Ricardo Martino Alba \\ Richard Bingham \\ Richard Cawley Madden \\ Richard Hain \\ Robert Bransfield \\ Robert J. Donovan \\ Robyn Fivush \\ Rodolfo Rocha Vieira Leocádio \\ Rodrigo Poblete Umanzor \\ Roger E. Thomas \\ Rohail Hassan \\ Rosalie Greenberg \\ Rose Martini \\ Rosemary Hiscock \\ Roslyn Livingstone \\ Roy McConkey \\ Rozeta Sokou \\ Ruchi Gera \\ Rüdiger Klapdor \\ Ruxandra Ioana Curea-Pitorac \\ Ryan D. Williamson \\ Ryan Rego \\ Sabino De Gisi \\ Salome Adam \\ Salvador Cruz Rambaud \\ Sam Telford \\ Sampath Parthasarathy \\ Samuel Asumadu-Sarkodie \\ Samuel N. Breit \\ Sara Campagna \\ Sara McNeillis
}

Sarah Prior

Satoru Yamada

Scott R. Sanders

Sema K. Aydede

Sharanjot Saini

Sharlene L. Gomes

Shatadru Chakravarty

Shayu Deshpande

Shervin Assari

Shervin Assari

Shervin Minaee

Shikha Saini

Shinichiro Ochi

Shino Oba

Shiqian Sherry Gao

Shuo Zhao

Simon Evans

Simone Aquino

Simone Garzon

Sitalakshmi Venkatraman

SoJung Kim

Sou Hyun Jang

Stefan Nilsson

Stephanie Worrell

Stergios Boussios

Steven G. Koven

Sudheer Ravuri

Sudipta Rakshit

Susan Cork

Susan Madison-Antenucci

Susanna McColley

Takashi Isezaki

Takatoshi Hara

Tan Boon Yeow

Tanisha Jowsey

Tatiana Christides

Tatsuya Kondo

Teresa Lesiuk

Tetsuya Homma

Thomas Wan

Tim Kilner

Titto T Idicula

Tobias Kutzner 


Tomoki Nakamura
Tsering Stobdan
U Rajendra Acharya
Umar Shafique
Usama Awan
Usha Sambamoorthi
Vaibhav Jain
Valentina Gatteschi
Valerie McLaughlin Crabtree
Valerio Pazienza
Venu Velagapudi
Vett Lloyd
Vickie Hughes
Vinay Jasani
Vincenzo Natale
Vivian Kjelland
Vivian Valdmanis
Vivienne Tippett
Viviette Allen
Wei-Hong Zhang
Wen Lin
Wendy A. Stewart
Wen-Wei Chang
Wilf McSherry
Wilson Abreu
Wim Van Den Noortgate
Vang

Wolfgang Leister

Wolfgang Rascher

Won Lee

Wui-Chiang Lee

Xerxes Tesoro Seposo

Xuan Hui

Ya-Wen Lee

Yet Hong Khor

Yigang Wei

Yinfeng Zhang

Yingjie Sun

Yongjae Yoo

Yoshihiro Ikura

Yubing Shi

YuChun Yao

Yuelong Ji

Yuhi Fujimoto

Yulia Solovieva

Yuliya Mysyuk

Yung Yau

Yuriy Bilan

Yu-Tung Huang

Zahra Jalal

Zakir Uddin

Zhaowei Kong

Zoe Kopsaftis

(C) 2020 by the author. Licensee MDPI, Basel, Switzerland. This article is an open access article distributed under the terms and conditions of the Creative Commons Attribution (CC BY) license (http://creativecommons.org/licenses/by/4.0/). 